\title{
Review of: "The perceived risk of being infected at work: An application of the job demands-resources model to workplace safety during the COVID-19 outbreak"
}

\section{Lara Bertola}

Potential competing interests: The author(s) declared that no potential competing interests exist.

\section{Comments to the Author}

This is a valuable study, however, in order to improve the quality of the article, you need to solve some existing problems.

1.The gap of knowledge is not clear enough to show the value and innovation of this study.

2. Please specify how the sample size is calculated.

3. What seems to me to be most appropriate is a discussion of the specific relevance of the science to your project. As written, the discussion is inherently too didactic to keep readers engaged, at least by my read.

4. The conclusion part should should be explored further 